\title{
Bloqueo de rama izquierda como equivalente ST, ¿cuándo sí, cuándo no?
}

\section{Left bundle branch block as equivalent of ST-segment elevation myocardial infarction: when yes, when not?}

\section{Laura Ceballos-Naranjo ${ }^{1}$ y Jonathan Cardona-Vélez ${ }^{2 *}$}

${ }^{1}$ Facultad de Medicina, Universidad Pontificia Bolivariana; ${ }^{2}$ Medicina Interna, Universidad de Antioquia, Hospital Universitario San Vicente Fundación, Medellín, Colombia

\section{Resumen}

La presencia de un bloqueo de rama izquierda del haz de His nuevo o presumiblemente nuevo junto con síntomas isquémicos se ha considerado tradicionalmente un equivalente electrocardiográfico de infarto agudo de miocardio con elevación del segmento ST, el cual debe ser llevado a reperfusión emergente. Para su definición se han propuesto varios criterios, pero ninguno ha alcanzado un rendimiento diagnóstico óptimo. A continuación detallaremos dichos criterios, sus principales problemas y las ventajas que han demostrado.

Palabras clave: Electrocardiografía. Infarto agudo de miocardio con elevación del segmento ST. Bloqueo de rama izquierda. Medicina interna. Cardiología. Colombia.

\begin{abstract}
A new or presumably new left bundle branch block along with ischemic symptoms has traditionally been considered an electrocardiographic equivalent of ST-segment elevation myocardial infarction, which should be brought to emergent reperfusion. However, several criteria have been proposed for its definition, but none has reached out an optimal diagnostic yield. Below we detail these criteria, their main problems and the advantages they have shown.
\end{abstract}

Key words: Electrocardiography. ST elevation myocardial infarction. Left bundle branch block. Internal medicine. Cardiology. Colombia.

\section{Correspondencia:}

*Jonathan Cardona-Vélez

E-mail: jcardonavelez@ hotmail.com
Fecha de recepción: 23-01-2018

Fecha de aceptación: 08-08-2018

DOI: 10.24875/ACM.M19000011
Disponible en internet: 19-03-2019 Arch Cardiol Mex. 2019;89(1):25-30 www.archivoscardiologia.com 1405-9940 @ 2019 Instituto Nacional de Cardiología Ignacio Chávez. Publicado por Permanyer México SA de CV. Este es un artículo Open Access bajo la licencia CC BY-NC-ND (http://creativecommons.org/licenses/by-nc-nd/4.0/). 


\section{Introducción}

El electrocardiograma (ECG) es una herramienta determinante en la evaluación de pacientes con dolor precordial, ya que permite establecer el diagnóstico de infarto agudo de miocardio (IAM), especialmente en aquellos con elevación del segmento ST (IAMST); sin embargo, en presencia de un bloqueo de rama izquierda del haz de His (BRIHH), las anormalidades de la repolarización modifican la morfología de las ondas y dificultan su lectura ${ }^{1}$.

En vista de estas consideraciones, para facilitar el diagnóstico de IAM en presencia de BRIHH se han propuesto varios criterios, pero ninguno ha alcanzado un rendimiento diagnóstico óptimo². Sgarbossa, et al. fueron los primeros en proponer una puntuación electrocardiográfica para el diagnóstico de IAM en presencia de esta entidad ${ }^{3}$; no obstante, aunque tiene una alta especificidad, la sensibilidad sigue siendo muy baja. Posteriormente, Smith, et al. propusieron una modificación a dichos criterios que, según sus resultados, aumentan de manera significativa la sensibilidad ${ }^{4}$.

\section{¿Bloqueo de rama izquierda como equivalente ST?}

En el contexto de esta incertidumbre diagnóstica, la presencia de un BRIHH nuevo o presumiblemente nuevo junto con síntomas isquémicos se ha considerado tradicionalmente un equivalente electrocardiográfico de IAMST, el cual debe ser llevado a reperfusión emergente ${ }^{2}$; sin embargo, recientes estudios han demostrado una baja incidencia de IAM en pacientes con BRIHH ${ }^{5,6}$. Debido a dichos hallazgos, las guías de tratamiento cambiaron radicalmente después de 20 años.

Las guías de la American Heart Association (AHA) de 1996 y 20047,8 recomendaban la terapia de reperfusión inmediata en pacientes con BRIHH nuevo o presumiblemente nuevo que presentaran síntomas isquémicos, basándose en un metaanálisis que demostró que la fibrinólisis se asociaba a un aumento en la supervivencia de los pacientes con BRIHH e IAM ${ }^{9}$; sin embargo, esta recomendación fue abolida en las guías del $2013^{10}$. Este cambio reduce la incidencia de una terapia fibrinolítica innecesaria que pueda generar hemorragias, principalmente en población de alto riesgo; aunque no realizar una terapia de reperfusión adecuada en pacientes con una verdadera oclusión arterial coronaria puede aumentar la morbilidad y mortalidad en esta población'.

En 2012, las guías de IAMST de la Sociedad Europea de Cardiología (SEC) y en 2014, las guías de revascularización miocárdica de la Sociedad Europea de Cirugía Cardiotorácica, recomendaron la realización de intervención coronaria percutánea (ICP) 0 reperfusión farmacológica para pacientes con síntomas isquémicos que presentaran $\mathrm{BRIHH}$ nuevo o presumiblemente nuevo en el ECG ${ }^{11,12}$. En el 2017 se publicaron las últimas guías de IAMST de la SEC, donde no solo siguen haciendo la misma recomendación, sino que también sugieren el uso de los criterios

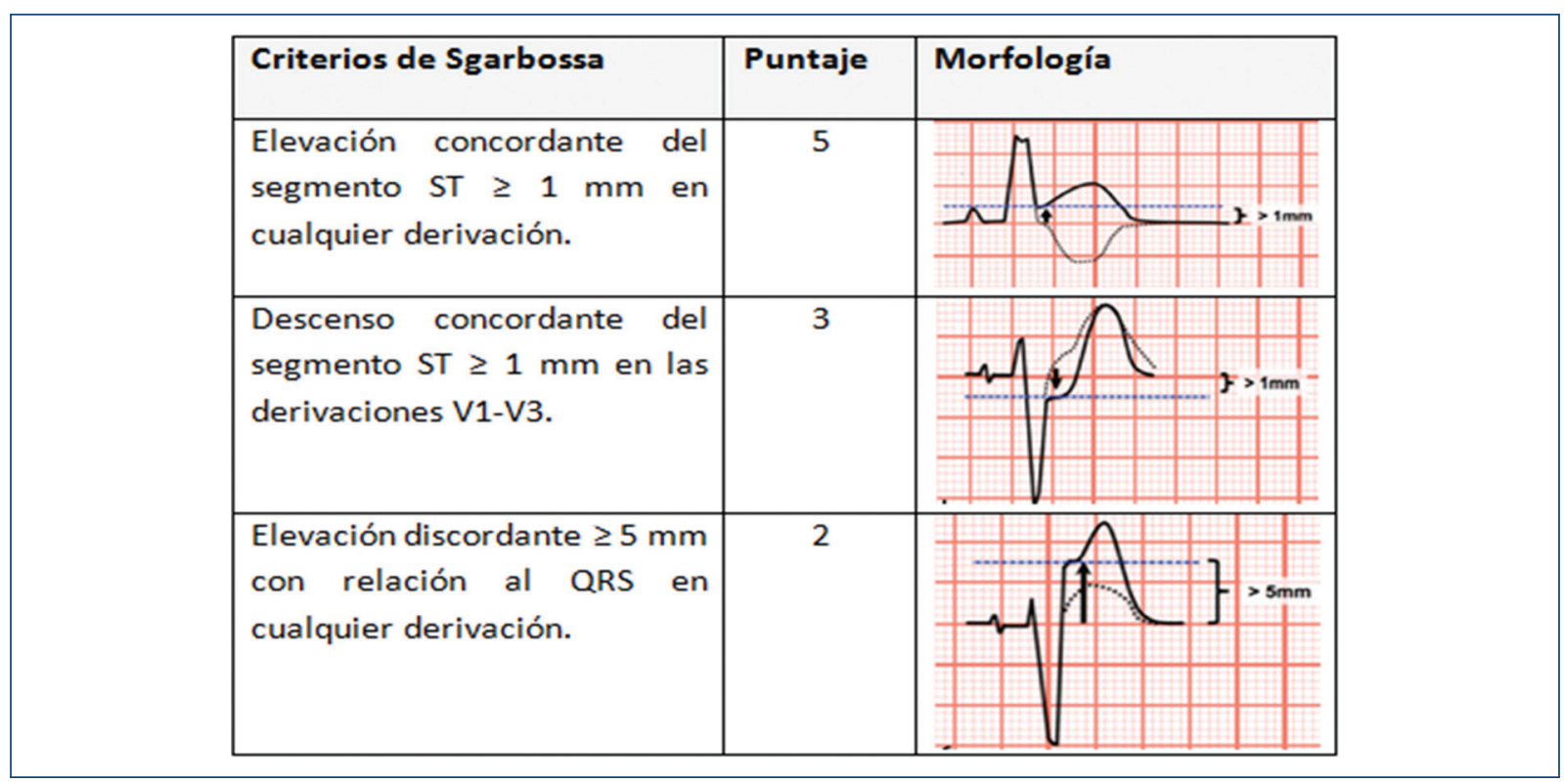

Figura 1. Criterios de Sgarbossa para el diagnóstico de IAMST en presencia de BRIHH. Un puntaje menor de 3 no es considerado tradicionalmente diagnóstico de IAMST, pero no lo descarta. 
de Sgarbossa, haciendo énfasis en que la elevación concordante del segmento ST es uno de los mejores indicadores de $\mathrm{IAM}^{13}$.

En 1996, Sgarbossa, et al. publicaron sus criterios a partir de un subanálisis del estudio GUSTO $^{3}$, en el cual el $0.6 \%$ de los pacientes con IAM tenían BRIHH. Los criterios de Sgarbossa (Fig. 1) se basan en un sistema de puntos en el cual se requieren tres o más para el diagnóstico de IAM en presencia de BRIHH, típicamente conocidos como criterios weighted. También se pueden utilizar los criterios sin dicho sistema de puntos, en este caso se acepta cualquiera de los tres, forma conocida como unweighted, la cual es más sensible pero menos específica ${ }^{4,14}$.

Estos criterios contribuyeron en gran medida a solucionar múltiples problemas a la hora de enfrentarse a un ECG con BRIHH en pacientes con síntomas isquémicos; sin embargo, el estudio tuvo dos limitaciones importantes ${ }^{1,4}$, por lo que no es sorprendente que los intentos de validar los criterios electrocardiográficos hayan mostrado una especificidad útil pero una sensibilidad deficiente:

- El estudio usó como desenlace de IAM la elevación de la creatincinasa $\mathrm{MB}(\mathrm{CK}-\mathrm{MB})$ en vez de usar la evidencia angiográfica de oclusión coronaria aguda, lo cual limita la sensibilidad de la regla, ya que combina el IAMST y el IAM no ST en la definición de su desenlace.

- Sgarbossa, et al. utilizaron un límite absoluto de $5 \mathrm{~mm}$ para la elevación discordante del segmento $\mathrm{ST}$, en lugar de un criterio proporcional que la relacione con el voltaje del QRS o S que la precede, lo cual podría ser más útil en términos de sensibilidad y especificidad ${ }^{1,3,4}$.

Aunque los criterios de Sgarbossa son un avance importante, tienen la limitación de su baja sensibilidad. En un metaanálisis se observó una sensibilidad general del $20 \%$ y una especificidad del $96 \%$ para una puntuación de Sgarbossa mayor o igual a tres $\mathrm{s}^{14,15}$. No obstante, la combinación de los dos primeros criterios (elevación concordante del segmento ST en cualquier derivada y descenso concordante del segmento ST en V1-V3) contribuye al aumento de la sensibilidad y la especificidad en un 37.2 y un $96.6 \%$ respectivamente ${ }^{16}$. Hallazgos similares han sido encontrados en múltiples estudios donde se ha demostrado que la elevación o depresión concordante del segmento ST son un predictor independiente de $\operatorname{IAM}^{17}$ y que la elevación concordante del segmento ST es el criterio individual más específico para el diagnóstico de IAM en presencia de $\mathrm{BRIHH}^{18}$.

Aproximadamente nueve años después del emblemático estudio de Sgarbossa, et al. un reporte de los investigadores del HERO-2 (Hirulog and early reperfusion or occlusion trial) comparó los tres criterios de Sgarbossa y analizó el valor pronóstico a 30 días. En el estudio original HERO-2 había 300 pacientes que presentaban síntomas isquémicos (más de 30 minutos de angina) y que tenían BRIHH nuevo o presumiblemente nuevo, estos se compararon con un grupo control de 300 pacientes que no presentaban $\mathrm{BRIHH}$ en el ECG. De los 300 pacientes con BRIHH, 92 cumplieron los criterios establecidos por Sgarbossa, et al. ${ }^{19}$.

El IAM, según lo determinaron los niveles de creatincinasa, se produjo en el $80.7 \%$ de los pacientes con BRIHH y en el $88.7 \%$ de los controles $(p=0.006)$. Los cambios del segmento ST fueron específicos pero no sensibles para el diagnóstico de IAM. Adicionalmente la mortalidad fue mayor en los pacientes con BRIHH. El estudio confirmó lo siguiente ${ }^{19}$ :

1. La elevación concordante del segmento ST en cualquier derivada de más de $1 \mathrm{~mm}$ tiene alta especificidad (98.3\%) pero baja sensibilidad (33.5\%) para IAM.

2. El descenso concordante del segmento ST de más de $1 \mathrm{~mm}$ en las derivadas V1 a V3 tiene alta especificidad, sin embargo la sensibilidad fue muy baja también (14.1\%).

3. La elevación discordante del segmento ST de más de $5 \mathrm{~mm}$ no fue sensible $(29.3 \%)$ ni específica (58.6\%) para el diagnóstico de IAM.

La baja utilidad del tercer criterio de Sgarbossa (elevación discordante mayor a $5 \mathrm{~mm}$ con relación al QRS en cualquier derivación) se debe a que no se considera la relación existente entre la elevación del segmento ST con el voltaje del QRS que lo precede (relación ST/S), concepto conocido como proporcionalidad ${ }^{14}$; esto corresponde a la relación obtenida entre la amplitud de la elevación discordante del segmento ST medida desde el punto $\mathrm{J}$ en precordiales ${ }^{4}$ con relación a la amplitud del QRS anterior (Fig. 2) ${ }^{1,20}$. En otras palabras, esta norma se basa en que la repolarización es proporcional a la despolarización, concepto fundamental para el diagnóstico del IAMST en el escenario de anormalidades morfológicas presentes en bloqueos de rama o hipertrofias ventriculares ${ }^{1,21}$.

Smith, et al. fueron los primeros en evaluar los algoritmos de Sgarbossa usando la ICP en la definición de su desenlace ${ }^{4,22}$ y en un intento por mejorar la sensibilidad reemplazaron el tercer criterio por una relación estadística. En este estudio de casos y controles, donde se analizaron pacientes con BRIHH y oclusión coronaria aguda, tuvo mejores resultados la implementación de los dos criterios de concordancia junto con una relación $\mathrm{ST} / \mathrm{S} \leq-0.25$, presentando una sensibilidad del $91 \%$, especificidad del $90 \%$ y LR+ 9, LR- 0.14,14,16. 


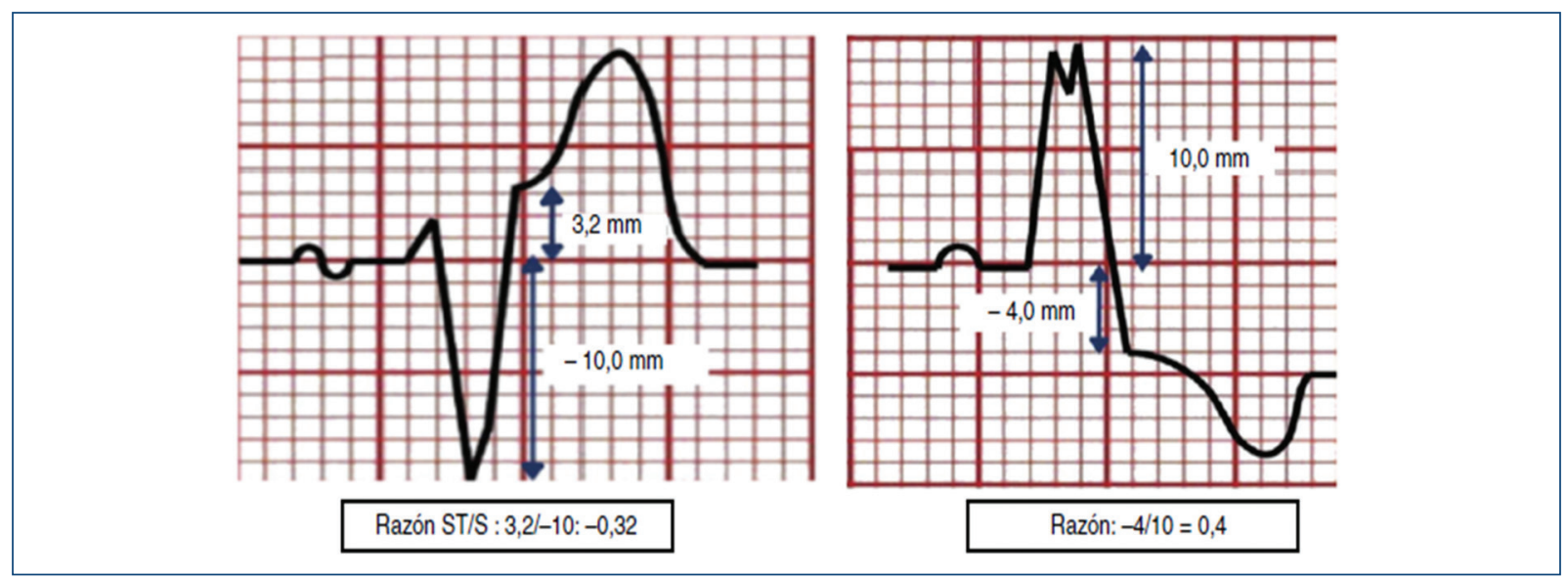

Figura 2. Criterio electrocardiográfico de proporcionalidad: cálculo de la relación ST/S (adaptado de Cai, et al. 2013)²3.

Tabla 1. Reglas de Smith para el diagnóstico de IAMST en presencia de BRIHH

\begin{tabular}{|c|c|}
\hline Reglas de Smith & Descripción \\
\hline $\begin{array}{l}\text { Regla I de Smith: puntuación de } \\
\text { Sgarbossa } \geq 3\end{array}$ & $\begin{array}{l}\text { Elevación concordante del } \mathrm{ST} \geq 1 \mathrm{~mm} \text { o depresión concordante del ST } \geq 1 \mathrm{~mm} \text { en las derivadas } \\
\text { V1-V3 }\end{array}$ \\
\hline $\begin{array}{l}\text { Regla II de Smith: puntuación de } \\
\text { Sgarbossa } \geq 2\end{array}$ & Puntuación de Sgarbossa $\geq 3$ o elevación discordante del ST $\geq 5 \mathrm{~mm}$ \\
\hline Regla III de Smith & Puntuación de Sgarbossa $\geq 3$ o elevación discordante del ST con una relación ST/S $\leq-0.25$ \\
\hline Regla IV de Smith & Puntuación de Sgarbossa $\geq 3$ o elevación discordante del ST con una relación ST/S $\leq-0.3$ \\
\hline Regla V de Smith & Desviación discordante del ST con un cociente $\mathrm{ST} / \mathrm{S}$ o $\mathrm{ST} / \mathrm{R} \leq-0.3$ \\
\hline
\end{tabular}

Las reglas I y || de Smith corresponden a los criterios de Sgarbossa con y sin el sistema de puntos respectivamente; siendo necesario para el diagnóstico de IAMST una puntuación de tres o más para la regla I y de dos o más para la regla II. La regla III representa una modificación de la regla II, donde se reemplaza el uso de una medida absoluta por una medida relativa de discordancia. La regla IV consiste en realizar una medida relativa de la desviación discordante, ya sea elevación o descenso del segmento ST, en lugar de una discordancia absoluta de la elevación del segmento ST. Por último, la regla $\checkmark$ se basa en una discordancia proporcional, ya sea por elevación o descenso del segmento ST (Tabla 1) ${ }^{4}$.

En un estudio realizado por Meyers HP, et al. se encontró que las reglas de Smith eran significativamente más sensibles que los criterios de Sgarbossa'. Di Marco $A$, et al. tuvieron resultados similares; en su estudio se incluyeron 145 pacientes, de los cuales 37 presentaban un cuadro clínico correspondiente a IAMST. Ellos demostraron que las reglas III y IV de Smith presentaron mejores resultados que los criterios de Sgarbossa y tuvieron una buena especificidad, del 90 y $97 \%$ respectivamente; sin embargo, la sensibilidad fue del $67 \%$ para la regla III de Smith y del $54 \%$ para la regla IV, la cual sigue siendo subóptima ${ }^{22}$.

A pesar de que las reglas de Smith mejoraron significativamente la sensibilidad con respecto a los criterios de Sgarbossa para el diagnóstico de IAMST en presencia de $\mathrm{BRIHH}$, poseen una limitación importante relacionada con la dificultad de su implementación en la práctica clínica. Cai, et al. propusieron un algoritmo diagnóstico simplificado que facilita su uso de manera sistemática, el cual incluye la implementación de ayudas diagnósticas adicionales que favorecen el abordaje de este tipo de pacientes (Fig. 3$)^{23}$.

\section{Conclusión}

La definición electrocardiográfica del IAMST en presencia de BRIHH continúa siendo todo un reto a pesar de los múltiples intentos por detallar con precisión esta condición. No en vano, las grandes sociedades científicas 


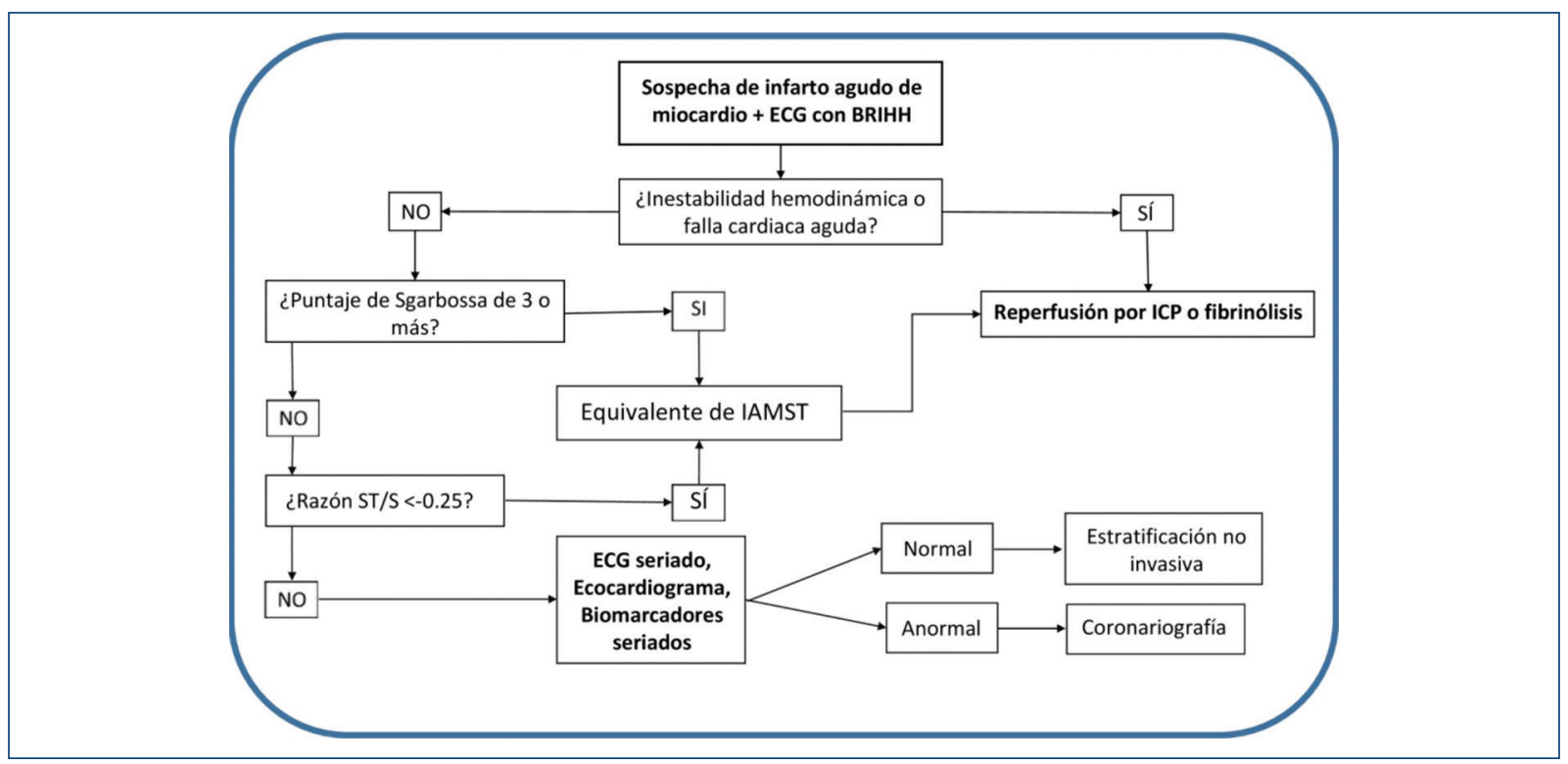

Figura 3. Algoritmo simplificado para el diagnóstico de IAMST en presencia de BRIHH (adaptado de Cai, et al. 201323. ICP: Intervención coronaria percutánea.

mundiales dan recomendaciones distintas. La última guía para el tratamiento del IAMST de la SEC 2017 es la única hasta ahora en recomendar el uso de criterios electrocardiográficos para facilitar el diagnóstico de IAM en presencia de BRIHH y en considerarlo como equivalente ST; sin embargo, ¿por qué si se ha demostrado que las reglas de Smith son más sensibles y específicas recomiendan el uso de los criterios de Sgarbossa?

La respuesta podría estar asociada a la facilidad en su aplicación respecto a las reglas de Smith, las cuales a pesar de ser prometedoras todavía cuentan con una sensibilidad deficiente.

Son aún muchas las preguntas por responder que hacen que el tratamiento apropiado de los pacientes con sospecha de IAM en presencia de BRIHH siga siendo controvertido y desafiante, motivo por el cual se precisa continuar en la búsqueda de criterios electrocardiográficos que permitan identificar aquellos pacientes que realmente necesitan una reperfusión emergente y evitar la realización de procedimientos innecesarios que podrían generar mayor morbilidad y mortalidad. Asimismo, el uso de herramientas complementarias al ECG como el ecocardiograma y los biomarcadores pueden brindar información útil en dilemas diagnósticos y terapéuticos.

\section{Conflicto de intereses}

Los autores declaran no tener conflicto de intereses ni haber recibido financiación.

\section{Responsabilidades éticas}

\section{Protección de personas y animales}

Los autores declaran que para esta investigación no se han realizado experimentos en seres humanos ni en animales.

\section{Confidencialidad de los datos}

Los autores declaran que han seguido los protocolos de su centro de trabajo sobre la publicación de datos de pacientes.

\section{Derecho a la privacidad y consentimiento informado}

Los autores declaran que en este artículo no aparecen datos de pacientes.

\section{Bibliografía}

1. Meyers HP, Limkakeng AT Jr, Jaffa EJ, Patel A, Theiling BJ, Rezaie SR, et al. Validation of the modified Sgarbossa criteria for acute coronary occlusion in the setting of left bundle branch block: A retrospective case-control study. Am Heart J. 2015;170(6):1255-64.

2. Ciliberti G, Del Pinto M, Notaristefano F, Zingarini G, Ambrosio G, Cavallini $C$. Left bundle branch block, chest pain and catheterization laboratory activation: an unavoidable cascade reaction? J Electrocardiol. 2016:49(4):504-8.

3. Sgarbossa EB, Pinski SL, Barbagelata A, Underwood DA, Gates KB, Topol EJ, et al. Electrocardiographic diagnosis of evolving acute myocardial infarction in the presence of left bundle-branch block. GUSTO-1 (Global Utilization of Streptokinase and Tissue Plasminogen Activator for Occluded Coronary Arteries) Investigators. N Engl J Med. 1996; $334: 481-7$. 
4. Smith SW, Dodd KW, Henry TD, Dvorak DM, Pearce LA. Diagnosis of ST-elevation myocardial infarction in the presence of left bundle branch block with the ST-elevation to S-wave ratio in a modified Sgarbossa rule. Ann Emerg Med. 2012:60:766-76.

5. Becker S, Chisholm G, Maeng M. Positive predictive value of clinically suspected ST-segment elevation myocardial infarction using angiographic verification. Am J Cardiol. 2013;112:923-7.

6. Neeland I, Kontos M, Lemos J. Evolving considerations in the management of patients with left bundle branch block and suspected myocardial infarction. J Am Coll Cardiol. 2012;60:96-105.

7. Ryan TJ, Anderson JL, Antman EM, Braniff BA, Brooks NH, Califf RM, et al. ACC/AHA guidelines for the management of patients with acute myocardial infarction. A report of the American College of Cardiology/ American Heart Association Task Force on Practice Guidelines (Committee on Management of Acute Myocardial Infarction). J Am Coll Cardiol. 1996;28(5):1328-428.

8. Antman EM, Anbe DT, Armstrong PW, Bates ER, Green LA, Hand M, et al. ACC/AHA guidelines for the management of patients with ST-elevation myocardial infarction: a report of the American College of Cardiology/American Heart Association Task Force on Practice Guidelines (Committee to Revise the 1999 Guidelines for the Management of Patients with Acute Myocardial Infarction). J Am Coll Cardiol. 2004; 44(3):E1-211.

9. Indications for fibrinolytic therapy in suspected acute myocardial infarction: Collaborative overview of early mortality and major morbidity results from all randomised trials of more than 1000 patients. Fibrinolytic Therapy Trialists' (FTT) Collaborative Group. Lancet. 1994;343(8893):311-22.

10. Anderson JL, Adams CD, Antman EM, Bridges CR, Califf RM, Casey DE Jr, et al. 2012 ACCF/AHA focused update incorporated into the ACCF/AHA 2007 guidelines for the management of patients with unstable angina/non-ST-elevation myocardial infarction: $A$ report of the American College of Cardiology Foundation/American Heart Association Task Force on Practice Guidelines. J Am Coll Cardiol. 2013; 61(23):e179-347.

11. Task Force on the management of ST-segment elevation acute myocardial infarction of the European Society of Cardiology (ESC), Steg PG James SK, Atar D, Badano LP, Blömstrom-Lundqvist C, Borger MA, et al. ESC Guidelines for the management of acute myocardial infarction in patients presenting with ST-segment elevation. Eur Heart J. 2012; 33(20):2569-619.

12. Authors/Task Force members, Windecker S, Kolh P, Alfonso F, Collet JP, Cremer J, Falk V, et al. 2014 ESC/EACTS Guidelines on myocardial revascularization: The Task Force on Myocardial Revascularization of the European Society of Cardiology (ESC) and the European Association for Cardio-Thoracic Surgery (EACTS). Developed with the special con- tribution of the European Association of Percutaneous Cardiovascular Interventions (EAPCI). Eur Heart J. 2014;35(37):2541-619.

13. Ibanez B, James S, Agewall S, Antunes MJ, Bucciarelli-Ducci C, Bueno H, et al. ESC Scientific Document Group. 2017 ESC Guidelines for the management of acute myocardial infarction in patients presenting with ST-segment elevation: The Task Force for the management of acute myocardial infarction in patients presenting with ST-segment elevation of the European Society of Cardiology (ESC). Eur Heart J. 2018;39(2):119-77

14. Herweg B, Marcus MB, Barold SS. Diagnosis of myocardial infarction and ischemia in the setting of bundle branch block and cardiac pacing. Herzschrittmacherther Elektrophysiol. 2016;27(3):307-22.

15. Tabas JA, Rodriguez RM, Seligman HK, Goldschlager NF. Electrocardiographic criteria for detecting acute myocardial infarction in patients with left bundle branch block: A meta-analysis. Ann Emerg Med. 2008;52(4):329-36.e1.

16. Moreno NL. Criterios de Sgarbossa: ¿es momento de actualizarlos? Rev Col Cardiol. 2015:22(6):294-7.

17. Kontos MC, Aziz HA, Chau VQ, Roberts CS, Ornato JP, Vetrovec GW Outcomes in patients with chronicity of left bundle-branch block with possible acute myocardial infarction. Am Heart J. 2011:161(4):698-704.

18. Jain S, Ting HT, Bell M, Bjerke CM, Lennon RJ, Gersh BJ, et al. Utility of left bundle branch block as a diagnostic criterion for acute myocardial infarction. Am J Cardiol. 2011;107(8):1111-6.

19. Wong CK, Stewart RA, Gao W, French JK, Raffel C, White HD. Prognostic differences between different types of bundle branch block during the early phase of acute myocardial infarction: insights from the Hirulog and Early Reperfusion or Occlusion (HERO)-2 trial. Eur Heart J. 2006;27(1):21-8.

20. Pera VK, Larson DM, Sharkey SW, Garberich RF, Solie CJ, Wang YL, et al. New or presumed new left bundle branch block in patients with suspected ST-elevation myocardial infarction. Eur Heart J Acute Cardiovasc Care. 2018;7(3):208-17.

21. Armstrong EJ, Kulkarni AR, Bhave PD, Hoffmayer KS, Macgregor JS, Stein JC, et al. Electrocardiographic criteria for ST-elevation myocardial infarction in patients with left ventricular hypertrophy. Am J Cardiol. 2012;110(7):977-83

22. Di Marco A, Anguera I, Rodríguez M, Sionis A, Bayes-Genis A, Rodríguez J, et al. Evaluación de los algoritmos de Smith para el diagnóstico de infarto aqudo de miocardio en presencia de bloqueo de rama izquierda del haz de His. Rev Esp Cardiol. 2017;70(7):559-66.

23. Cai Q, Mehta N, Sgarbossa EB, Pinski S, Wagner G, Califf R, et al. The left bundle-branch block puzzle in the 2013 ST-elevation myocardial infarction guideline: From falsely declaring emergency to denying reperfusion in a high-risk population. Are the Sgarbossa criteria ready for prime time? Am Heart J. 2013;166(3):409-13. 